\title{
Probing the parameter space for an MSSM inflation and the neutralino dark matter
}

\author{
Rouzbeh Allahverdi, ${ }^{1}$ Bhaskar Dutta, ${ }^{2}$ and Anupam Mazumdar ${ }^{3}$ \\ ${ }^{1}$ Perimeter Institute for Theoretical Physics, Waterloo, ON, N2L 2Y5, Canada \\ ${ }^{2}$ Department of Physics, Texas A\&M University, College Station, Texas 77843-4242, USA \\ ${ }^{3}$ NORDITA, Blegdamsvej-17, Copenhagen-2100, Denmark
}

(Received 19 February 2007; published 24 April 2007)

\begin{abstract}
The flat directions $L L e$ and $u d d$ within the minimal supersymmetric standard model provide all the necessary ingredients for a successful inflation with the right amplitude of the scalar density perturbations, negligible gravity waves and the spectral tilt within $2 \sigma$ observed range $0.92 \leq n_{s} \leq 1.0$. In this paper we explore the available parameter space for inflation in conjunction with a thermal cold dark matter abundance within the minimal supergravity model. Remarkably for the inflaton, which is a combination of squarks and sleptons, there is a stau-neutralino coannihilation region below the inflaton mass $500 \mathrm{GeV}$ for the observed density perturbations and the tilt of the spectrum. For such a low mass of the inflaton the LHC is capable of discovering the inflaton candidates within a short period of its operation. Inflation is also compatible with the focus point region which opens up for the inflaton masses above TeV. We show that embedding MSSM within $S O(10)$ can naturally favor this region.
\end{abstract}

DOI: 10.1103/PhysRevD.75.075018

PACS numbers: 12.60.Jv, 98.80.Cq

\section{INTRODUCTION}

The minimal supersymmetric standard model (MSSM) provides us all the necessary ingredients for a successful slow roll inflation which can explain the flatness, homogeneity and the isotropy problems of the hot big bang cosmology [1-5], for details see Ref. [4]. ${ }^{1}$ Not only that the inflaton carries the standard model (SM) charges, the candidates are the two flat directions within MSSM; LLe and $u d d$, where $L$ stands for the superfields corresponding to lepton doublets, while $u, d$ and $e$ are the right-handed components of (up and down type) squarks and selectron, respectively. $^{2}$

Moreover the biggest advantage of having an MSSM inflaton is the predictivity, the model parameters (the mass and the couplings) will hopefully be discovered at the LHC [8]. The MSSM inflaton is robust ${ }^{3}$ in its prediction on the cosmic microwave background (CMB) radiation; the model yields the right amplitude for the scalar density perturbations, the scalar spectral index lies within the allowed range $0.92 \leq n_{s} \leq 1.0[9,10]$, which is within the $2 \sigma$ error bar. The model does not predict any observable gravity waves in concurrence to the CMB observations nor does it produce any significant non-Gaussianity.

\footnotetext{
${ }^{1}$ Those readers who wish to seek models of an observable universe without invoking inflation, see Ref. [6].

${ }^{2}$ This is the first realistic example of the inflaton where it carries the SM charges, all other attempts failed in the past [7]. Another gauge-invariant candidate for the inflaton, $\mathrm{NH}_{u} \mathrm{~L}$ with the neutrino $N$ being Dirac, has also been proposed in Ref. [2]. This model has a similar prediction to that of LLe and $u d d$, but relies solely on the renormalizable interactions. In Ref. [5] we explored the possibility of a gauge-invariant inflaton within gauge mediated supersymmetry breaking.

${ }^{3}$ Furthermore the model does not suffer from supergravity (SUGRA) and trans-Planckian corrections [1,4]. The well known $\eta$ problem is absent.
}

The end of inflation marks the coherent oscillations of the inflaton and subsequent decay of the flat direction. The decay products are distinctively MSSM degrees of freedom by virtue of the inflaton's gauge couplings to the SM quarks/squarks and gauge bosons/gauginos. The MSSM degrees of freedom reheat the Universe to a thermal bath at a temperature above the $\mathrm{TeV}$ scale. ${ }^{4}$ Such a reheat temperature is sufficient to answer two of the outstanding puzzles; thermal production of cold dark matter (CDM) and baryon asymmetry via electroweak baryogenesis within MSSM [4].

It is well known that the lightest supersymmetric particle (LSP) is absolutely stable and can be a candidate for the CDM. In most models the lightest neutralino is mostly bino (the superpartner of the hypercharge gauge boson), with negligible admixture of wino and/or Higgsino. It is then a natural question to ask what is the overlapping parameter region of MSSM which would predict a successful inflation and also thermally generated neutralino. ${ }^{5}$

At the grand unified (GUT) scale there is a particular ansatz for the soft supersymmetry (SUSY) breaking parameters which is very well motivated in the literature known as the "mSUGRA" (Constrained MSSM) [12,13], it assumes that all of the squark and slepton soft masses are the same at the GUT scale to suppress flavor violation. Similarly all of the $A$-terms are also taken to be flavor independent and universal at the GUT scale as well. Finally all of the gaugino masses are taken to be the same at the GUT scale. With this ansatz, the parameters are RGE evolved to the $\mathrm{TeV}$ scale and masses and interactions of

\footnotetext{
${ }^{4}$ The final reheating is obtained only when all flat directions are completely evaporated [11].

${ }^{5}$ In models with gauge mediated SUSY breaking gravitino is the LSP. In Ref. [5], we have studied gravitino dark matter in conjunction with MSSM inflation in these models.
} 
the particles are studied. The recent dark matter constraints and other experimental results have separated this parameter space mostly into three basic regions: stau-neutralino coannihilation, $A$ annihilation, and focus point region [14]. The most interesting question is whether the allowed values of $m_{\phi}$ from the inflation constraints fall in any of these regions. Moreover, it is a burden on any inflationary model and the underlying theory to provide conditions for the observable CDM abundance and the candidate. ${ }^{6}$

In order to address these questions, we will first consider the observables and constraints from CMB and then use them to constrain the inflaton mass. We will then use this inflaton mass to investigate the mSUGRA parameter space for the allowed neutralino type dark matter. We will also discuss the flat directions when right-handed (RH) neutrinos are present in the model and the consequences of embedding MSSM in $S U(5)$ or $S O(10)$ models of grand unified theory (GUT).

The paper is organized as follows. In Sec. II, we review inflation in MSSM. In Sec. III, we discuss the constraints on the inflaton mass arising from spectral index and the amplitude of perturbations. In Sec. IV, we discuss the mSUGRA parameter space and the constraints arising from the allowed values of inflaton mass. In Sec. V, we discuss the flat directions and their lifting when RH neutrinos are added, and possible embedding in $S U(5)$ and $S O(10)$. Section VI contains our conclusions.

\section{A BRIEF REVIEW OF MSSM INFLATION}

\section{A. Inflation near a saddle point}

Let us recapitulate the main features of MSSM flat direction inflation $[1,4]$. In the limit of unbroken SUSY the flat directions have exactly vanishing potential. This situation changes when soft SUSY breaking and nonrenormalizable superpotential terms of the type [15]

$$
W_{\text {non }}=\sum_{n>3} \frac{\lambda_{n}}{n} \frac{\Phi^{n}}{M^{n-3}},
$$

are included. Here $\Phi$ is a gauge-invariant superfield which contains the flat direction. Within MSSM all the flat directions are lifted by nonrenormalizable operators with $4 \leq$ $n \leq 9$ [16], where $n$ depends on the flat direction. We expect that quantum gravity effects yield $M=M_{\mathrm{P}}=$ $2.4 \times 10^{18} \mathrm{GeV}$ and $\lambda_{n} \sim \mathcal{O}(1)$ [17].

Let us focus on the lowest order superpotential term in Eq. (1) which lifts the flat direction. Soft SUSY breaking induces a mass term and an $A$ term so that the scalar potential along the flat direction reads

\footnotetext{
${ }^{6}$ We notice that the low scale of MSSM inflation makes it difficult to invoke a late stage of entropy release which can dilute thermal overabundance of LSP (as it happens in the bulk region), or, produce nonthermal dark matter.
}

$$
V=\frac{1}{2} m_{\phi}^{2} \phi^{2}+A \cos \left(n \theta+\theta_{A}\right) \frac{\lambda_{n} \phi^{n}}{n M_{\mathrm{P}}^{n-3}}+\lambda_{n}^{2} \frac{\phi^{2(n-1)}}{M_{\mathrm{P}}^{2(n-3)}},
$$

Here $\phi$ and $\theta$ denote, respectively, the radial and the angular coordinates of the complex scalar field $\Phi=$ $\phi \exp [i \theta]$, while $\theta_{A}$ is the phase of the $A$-term (thus $A$ is a positive quantity with dimension of mass). The maximum impact from the $A$-term is obtained when $\cos (n \theta+$ $\left.\theta_{A}\right)=-1$ (which occurs for $n$ values of $\theta$ ).

In the gravity mediated SUSY breaking case, the $A$ term and the soft SUSY breaking mass terms are of the same order of magnitude as the gravitino mass, i.e. $m_{\phi} \sim A \sim$ $m_{3 / 2} \sim \mathcal{O}(1) \mathrm{TeV}$. Then, as pointed out in Ref. [1], if $A$ and $m_{\phi}$ are related by

$$
A^{2}=8(n-1) m_{\phi}^{2}
$$

there is a saddle point:

$$
\phi_{0}=\left(\frac{m_{\phi} M_{\mathrm{P}}^{n-3}}{\lambda_{n} \sqrt{2 n-2}}\right)^{1 /(n-2)},
$$

where $V^{\prime}(\phi)=V^{\prime \prime}\left(\phi_{0}\right)=0$. The potential is very flat near $\phi_{0}$, and it is given by

$$
V_{0}=\frac{(n-2)^{2}}{2 n(n-1)} m_{\phi}^{2} \phi_{0}^{2} .
$$

As a result, if the flat direction is in the vicinity of $\phi_{0}$ (and has a sufficiently small kinetic energy), there will be an ensuing phase of inflation. ${ }^{7}$

The Hubble expansion rate during inflation is given by

$$
H_{\mathrm{inf}}=\frac{(n-2)}{\sqrt{6 n(n-1)}} \frac{m_{\phi} \phi_{0}}{M_{\mathrm{P}}} .
$$

Inflation ends when $|\eta| \sim 1$, where $\epsilon \equiv\left(M_{\mathrm{P}}^{2} / 2\right)\left(V^{\prime} / V\right)^{2}$ and $\eta \equiv M_{\mathrm{P}}^{2}\left(V^{\prime \prime} / V\right)$ are the slow roll parameters. The number of $e$ foldings between the time when the observationally relevant perturbations are generated and the end of inflation follows: $\mathcal{N}_{\mathrm{COBE}} \simeq 66.9+(1 / 4) \ln \left(V_{0} / M_{\mathrm{P}}^{4}\right)$ [19].

Here we have used the fact that, due to efficient reheating, the energy density in the inflaton gets converted into MSSM radiation very quickly after the end of MSSM inflation (for details see Ref. [4]). The amplitude of the perturbations thus produced is given by

\footnotetext{
${ }^{7}$ In Ref. [18] we have addressed how the flat direction end up at $\phi_{0}$. This is an initial condition problem which is addressed if there were prior phases of inflation. In the context of string theory where there are multiple false vacua below the string scale, it is conceivable that an eternal inflation is generic, however, a graceful exit of inflation must require a phase of MSSM inflation in the observable world to reheat the plasma with the desired SM degrees of freedom for the big bang nucleosynthesis [1-5]. In this regard a string landscape creates an ideal initial condition for the MSSM inflation.
} 


$$
\delta_{H} \simeq \frac{1}{5 \pi} \sqrt{\frac{2}{3} n(n-1)}(n-2)\left(\frac{m_{\phi} M_{\mathrm{P}}}{\phi_{0}^{2}}\right) \mathcal{N}_{\mathrm{COBE}}^{2} .
$$

The spectral index for the power spectrum is found to be [1]

$$
n_{s}=1-\frac{4}{\mathcal{N}_{\mathrm{COBE}}}
$$

For weak scale supersymmetry, acceptable $\delta_{H}$ and $n_{s}$ are obtained if $n=6$. In this case, see Eqs. (4) and (6), we will have $\phi_{0} \sim 10^{14} \mathrm{GeV}$ and $H_{\text {inf }} \sim \mathcal{O}(1 \mathrm{GeV})$. This singles out two flat directions as the inflaton candidate: LLe and $u d d$. From Eq. (5) it turns out that $\mathcal{N}_{\mathrm{COBE}} \sim 50$, implying that $n_{s} \simeq 0.92$. This is compatible within the fit from combined WMAP-3 and SDSS data within $2 \sigma[9,10]$, though it lies at the lower end.

\section{B. Deviation from saddle point}

Inflation can still happen for small deviations from the saddle point condition Eq. (3). To quantify this, we define a parameter $\alpha^{2}$ such that $[3,4]$

$$
\frac{A^{2}}{8(n-1) m_{\phi}^{2}} \equiv 1+\left(\frac{n-2}{2}\right)^{2} \alpha^{2} .
$$

For $\alpha^{2} \neq 0$, the saddle point becomes a point of inflection where $V^{\prime \prime}\left(\phi_{0}\right)=0$, and

$$
V^{\prime}\left(\phi_{0}\right)=\left(\frac{n-2}{2}\right)^{2} \alpha^{2} m_{\phi}^{2} \phi_{0} .
$$

If $\alpha^{2}<0$, the potential has a local minimum and a maximum. In this case the flat direction is trapped in the local minimum. It will eventually tunnel past the maximum and a period of slow roll inflation will follow [4]. If $\alpha^{2}>0$, the potential has no maximum or local minimum, and then slow roll inflation occurs around $\phi_{0}$.

For $\alpha^{2} \neq 0$ the expressions for $n_{s}$ and $\delta_{H}$ are modified as [20] (see also Ref. [3])

$$
\begin{aligned}
\delta_{H}= & \frac{1}{5 \pi} \sqrt{\frac{2}{3} n(n-1)}(n-2) \frac{m_{\phi} M_{\mathrm{P}}}{\phi_{0}^{2}} \\
& \times \frac{1}{\Delta^{2}} \sin ^{2}\left[\mathcal{N}_{\mathrm{COBE}} \sqrt{\Delta^{2}}\right],
\end{aligned}
$$

and

$$
n_{s}=1-4 \sqrt{\Delta^{2}} \cot \left[\mathcal{N}_{\mathrm{COBE}} \sqrt{\Delta^{2}}\right]
$$

where

$$
\Delta^{2} \equiv n^{2}(n-1)^{2} \alpha^{2} \mathcal{N}_{\text {COBE }}^{2}\left(\frac{M_{\mathrm{P}}}{\phi_{0}}\right)^{4}
$$

Note that for $\alpha^{2}=0$, Eqs. (11) and (12) are reduced to (7) and (8) respectively. For $\alpha^{2}<0$, the spectral index will be smaller than that in Eq. (8), thus outside the $2 \sigma$ region from observations. The more interesting case, as pointed out in
Ref. [3], happens for $\alpha^{2}>0$. We can in this case get all values within the allowed range $0.92 \leq n_{s} \leq 1$ [10] for

$$
0 \leq \Delta^{2} \leq \frac{\pi^{2}}{4 \mathcal{N}_{\mathrm{COBE}}^{2}} \text {. }
$$

\section{CONSTRAINTS ON THE INFLATON MASS}

The inflaton mass, $m_{\phi}$ is constrained by the experimental data on the spectral index $n_{s}[9,10]$ and $\delta_{H}$ [21].

We first find the solutions of $m_{\phi}$ by solving Eqs. (11) and (12). $n_{s}$ depends mainly on $\Delta^{2}$ and is mostly independent of $m_{\phi}$ and $\lambda$ [the coupling in Eq. (1)]. The parameter $\Delta^{2}$ is defined in Eq. (14). We therefore solve $\Delta^{2}$ from Eq. (12) and apply this solution to determine the bounds on $m_{\phi}$ from the Eq. (11). In Fig. 1, we show $n_{s}$ as a function of $\Delta^{2}$. The range for $\Delta^{2}$ is determined from Eq. (14).

In Fig. 2, we show $\delta_{H}$ as a function of $n_{s}$ for different values of $m_{\phi}$. The blue band shows the experimentally allowed region. We find that smaller values of $m_{\phi}$ are preferred for smaller values of $n_{s}$. We also find that the allowed range of $m_{\phi}$ is $75-440 \mathrm{GeV}$ for the experimental ranges of $n_{s}$ and $\delta_{H}$. We assume $\lambda \sim 1$ for these two figures. If $\lambda$ is less than $\mathcal{O}(1)$, e.g., $\lambda \sim 0.1$ or so (which can occur in $S O(10)$ model), it will lead to an increase in $m_{\phi}$. Now we need to study these allowed ranges of the inflaton mass in the mSUGRA model. Since the inflaton mass is related to the parameters of the mSUGRA model, the main question is whether the allowed range of the inflaton mass is consistent with the experimentally allowed mSUGRA model or not.

\section{INFLATION AND DARK MATTER IN MSUGRA}

Since $m_{\phi}$ is related to the scalar masses, sleptons ( $L L e$ direction) and squarks ( $u d d$ direction), the bound on $m_{\phi}$ will be translated into the bounds on these scalar masses which are expressed in terms of the model parameters [4].

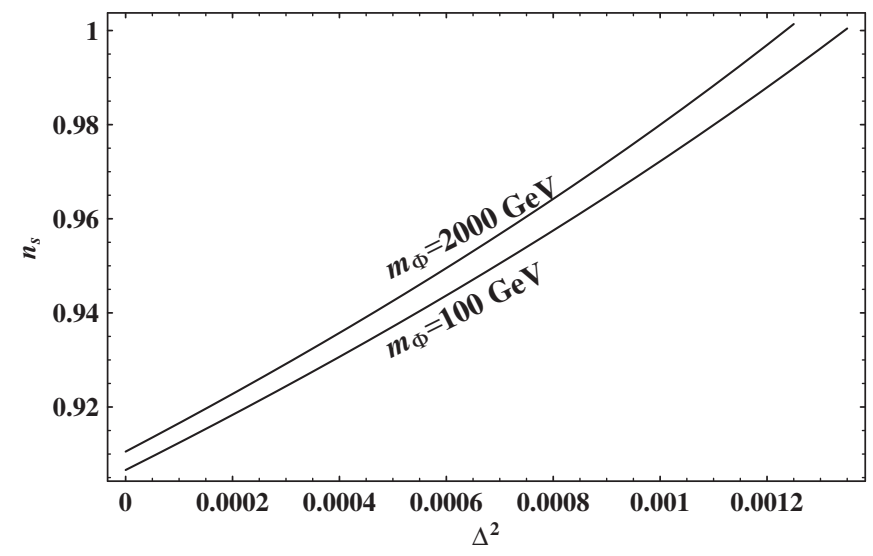

FIG. 1. $n_{s}$ is plotted as a function of $\Delta^{2}$ for different values of $m_{\phi} . \Delta$ is defined in the text. We choose $\lambda=1$. 


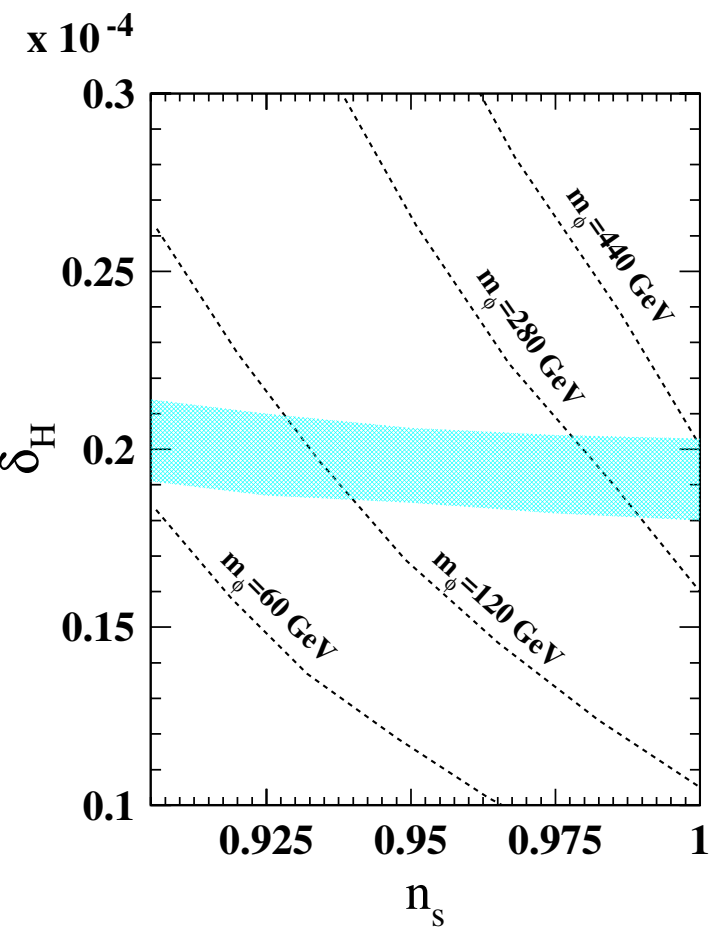

FIG. 2 (color online). $\delta_{H}$ is plotted as a function of $\Delta^{2}$ for different values of $m_{\phi}$. We used $\lambda=1$. The blue band denotes the experimentally allowed values of $\delta_{H}$.

The models of mSUGRA depend only on four parameters and one sign. These are $m_{0}$ (the universal scalar soft breaking mass at the GUT scale $M_{\mathrm{G}}$ ); $m_{1 / 2}$ (the universal gaugino soft breaking mass at $M_{\mathrm{G}}$ ); $A_{0}$ (the universal trilinear soft breaking mass at $\left.M_{\mathrm{G}}\right)^{8} ; \tan \beta=\left\langle H_{2}\right\rangle\left\langle H_{1}\right\rangle$ at the electroweak scale (where $H_{2}$ gives rise to $u$ quark masses and $H_{1}$ to $d$ quark and lepton masses); and the sign of $\mu$, the Higgs mixing parameter in the superpotential $\left(W_{\mu}=\right.$ $\left.\mu H_{1} H_{2}\right)$. Unification of gauge couplings within supersymmetry suggests that $M_{\mathrm{G}} \simeq 2 \times 10^{16} \mathrm{GeV}$. The model parameters are already significantly constrained by different experimental results. Most important constraints are:

(i) The light Higgs mass bound of $M_{h^{0}}>114.0 \mathrm{GeV}$ from LEP [22].

(ii) The $b \rightarrow s \gamma$ branching ratio [23]: $2.2 \times 10^{-4}<$ $\mathcal{B}\left(B \rightarrow X_{s} \gamma\right)<4.5 \times 10^{-4}$.

(iii) In mSUGRA the $\tilde{\chi}_{1}^{0}$ is the candidate for CDM. The $2 \sigma$ bound from the WMAP [9] gives a relic density bound for CDM to be $0.095<\Omega_{\mathrm{CDM}} h^{2}<0.129$.

(iv) The bound on the lightest chargino mass of $M_{\tilde{\chi}_{1}^{ \pm}}>$ $104 \mathrm{GeV}$ from LEP [24].

\footnotetext{
${ }^{8}$ The relationship between the two $A$ terms, the trilinear, $A_{0}$ and the nonrenormalizable $A$ term in Eq. (2) can be related to each other, however, that depends on the SUSY breaking sector. For a Polonyi model, they are given by $A=(3-\sqrt{3}) /(6-$ $\sqrt{3}) A_{0}$ [4]
}

(v) The possible $3.3 \sigma$ deviation (using $e^{+} e^{-}$data to calculate the leading order hadronic contribution) from the SM expectation of the anomalous muon magnetic moment from the muon $g-2$ Collaboration [25].

The allowed mSUGRA parameter space, at present, has mostly three distinct regions: (i) the stau-neutralino $\left(\tilde{\tau}_{1}-\right.$ $\tilde{\chi}_{0}^{1}$ ), coannihilation region where $\tilde{\chi}_{0}^{1}$ is the lightest SUSY particle (LSP), (ii) the $\tilde{\chi}_{0}^{1}$ having a dominant Higgsino component (focus point) and (iii) the scalar Higgs $\left(A^{0}, H^{0}\right)$ annihilation funnel $\left(2 M_{\tilde{\chi}_{0}^{1}} \simeq M_{A^{0}, H^{0}}\right)$. These three regions have been selected out by the CDM constraint. There stills exists a bulk region where none of these above properties is observed, but this region is now very small due to the existence of other experimental bounds. After considering all these bounds we will show that there exists an interesting overlap between the constraints from inflation and the CDM abundance.

We calculate $m_{\phi}$ at $\phi_{0}$ and $\phi_{0}$ is $10^{14} \mathrm{GeV}$ which is 2 orders of magnitude below the GUT scale. From this $m_{\phi}$, we determine $m_{0}$ and $m_{1 / 2}$ by solving the RGEs for fixed values of $A_{0}$ and $\tan \beta$. The RGEs for $m_{\phi}$ are

$$
\begin{aligned}
\mu \frac{d m_{\phi}^{2}}{d \mu} & =\frac{-1}{6 \pi^{2}}\left(\frac{3}{2} M_{2}^{2} g_{2}^{2}+\frac{9}{10} M_{1}^{2} g_{1}^{2}\right), & & (\text { for } L L e) \\
\mu \frac{d m_{\phi}^{2}}{d \mu} & =\frac{-1}{6 \pi^{2}}\left(4 M_{3}^{2} g_{3}^{2}+\frac{2}{5} M_{1}^{2} g_{1}^{2}\right), & & (\text { for } u d d) .
\end{aligned}
$$

$M_{1}, M_{2}$, and $M_{3}$ are $U(1), S U(2)$, and $S U(3)$ gaugino masses, respectively.

After we determine $m_{0}$ and $m_{1 / 2}$ from $m_{\phi}$, we can determine the allowed values of $m_{\phi}$ from the experimental bounds on the mSUGRA parameters space. In order to obtain the constraint on the mSUGRA parameter space, we calculate the SUSY particle masses by solving the RGEs at the weak scale using four parameters of the mSUGRA model and then use these masses to calculate Higgs mass, $\mathrm{BR}[b \rightarrow s \gamma]$, dark matter content, etc.

We show that the mSUGRA parameter space in Figs. 3 and 4 for $\tan \beta=10$ and 40 with the $u d d$ flat direction using $\lambda=1 .{ }^{9}$ In the figures, we show contours correspond to $n_{s}=1$ for the maximum value of $\delta_{H}=2.03 \times 10^{-5}$ (at $2 \sigma$ level) and $n_{s}=1.0,0.98,0.96$ for $\delta_{H}=1.91 \times 10^{-5}$. The constraints on the parameter space arising from the inflation appearing to be consistent with the constraints arising from the dark matter content of the universe and other experimental results. We find that $\tan \beta$ needs to be smaller to allow for smaller values of $n_{s}<1$. It is also interesting to note that the allowed region of $m_{\phi}$, as required by the inflation data for $\lambda=1$ lies in the stauneutralino coannihilation region which requires smaller

\footnotetext{
${ }^{9}$ We have a similar figure for the flat direction $L L e$ which we do not show in this paper. All the figures are for $u d d$ flat direction as an inflaton.
} 


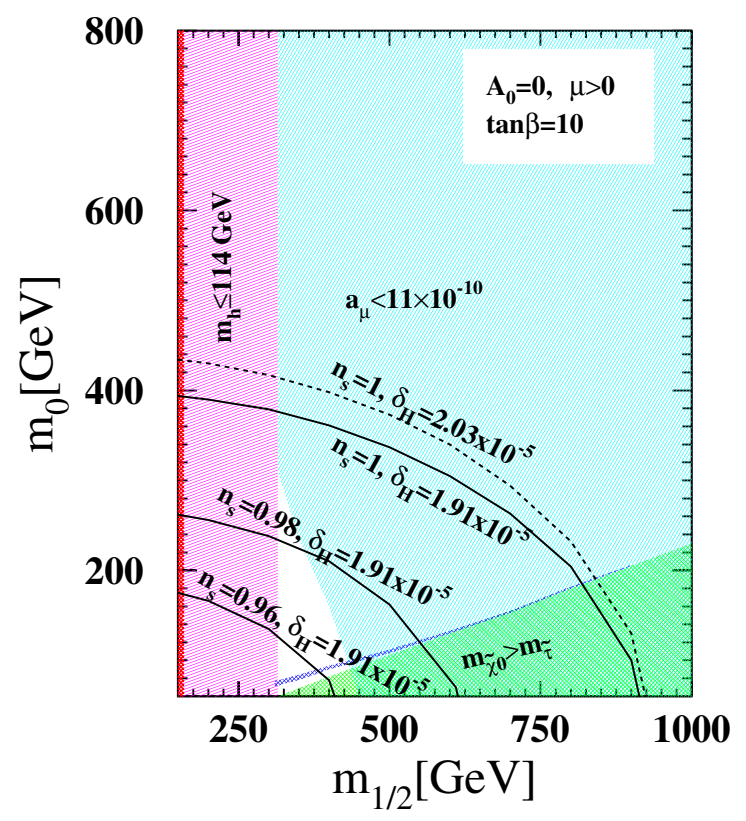

FIG. 3 (color online). The contours for different values of $n_{s}$ and $\delta_{H}$ are shown in the $m_{0}-m_{1 / 2}$ plane for $\tan \beta=10$. We used $\lambda=1$ for the contours. We show the dark matter allowed region narrow blue corridor, $(g-2)_{\mu}$ region (light blue) for $a_{\mu} \leq 11 \times 10^{-8}$, Higgs mass $\leq 114 \mathrm{GeV}$ (pink region) and LEPII bounds on SUSY masses (red). We also show the dark matter detection rate by vertical blue lines.

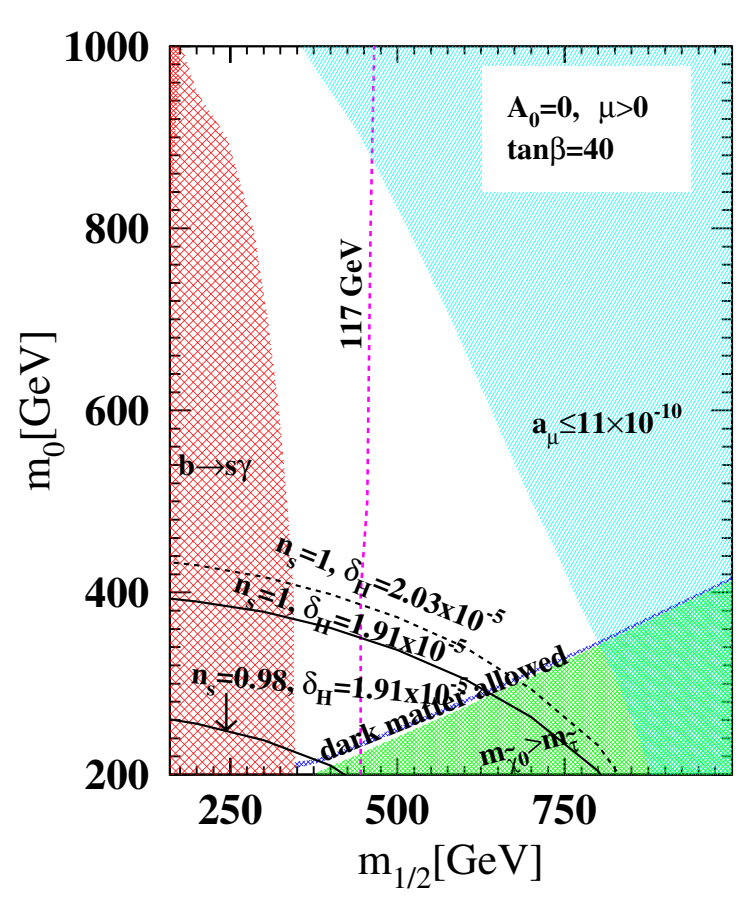

FIG. 4 (color online). The contours for different values of $n_{s}$ and $\delta_{H}$ are shown in the $m_{0}-m_{1 / 2}$ plane for $\tan \beta=40$. We used $\lambda=1$ for the contours. We show the dark matter allowed region narrow blue corridor, $(g-2)_{\mu}$ region (light blue) for $a_{\mu} \leq 11 \times 10^{-8}, b \rightarrow s \gamma$ allowed region (brick) and LEPII bounds on SUSY masses (red). values of the SUSY particle masses. The SUSY particles in this parameter space are, therefore, within the reach of the LHC very quickly. The detection of the region at the LHC has been considered in Ref. [26]. From the figures, one can also find that as $\tan \beta$ increases, the inflation data along with the dark matter, rare decay, and Higgs mass constraint allow smaller ranges of $m_{1 / 2}$. For example, the allowed ranges of gluino masses are $765 \mathrm{GeV}-2.1 \mathrm{TeV}$ and $900 \mathrm{GeV}-1.7 \mathrm{TeV}$ for $\tan \beta=10$ and 40 , respectively.

So far we have chosen $\lambda=1$. Now if $\lambda$ is small e.g., $\lambda \lesssim 10^{-1}$, we find that the allowed values of $m_{\phi}$ to be large. In this case the dark matter allowed region requires the lightest neutralino to have larger Higgsino component in the mSUGRA model. As we will see shortly, this small value of $\lambda$ is accommodated in $S O(10)$ type model. In Fig. 5, we show $n_{s}=1,0.98$ contours for $\delta_{H}=1.91 \times$ $10^{-5}$ in the mSUGRA parameter space for $\tan \beta=10$. In this figure, we find that $n_{s}$ can not smaller than 0.97, but if we lower $\lambda$ which will demand larger $m_{\phi}$ and therefore $n_{s}$ can be lowered down to 0.92 .

In Fig. 6, we show the contours of $\lambda$ for different values of $m_{\phi}$ which are allowed by $n_{s}$ and $\delta_{H}=1.91 \times 10^{-3}$. The blue bands show the dark matter allowed regions for $\tan \beta=10$. The band on the left is due to the stauneutralino coannihilation region allowed by other constraints and the allowed values of $\lambda$ are $0.3-1$. The first

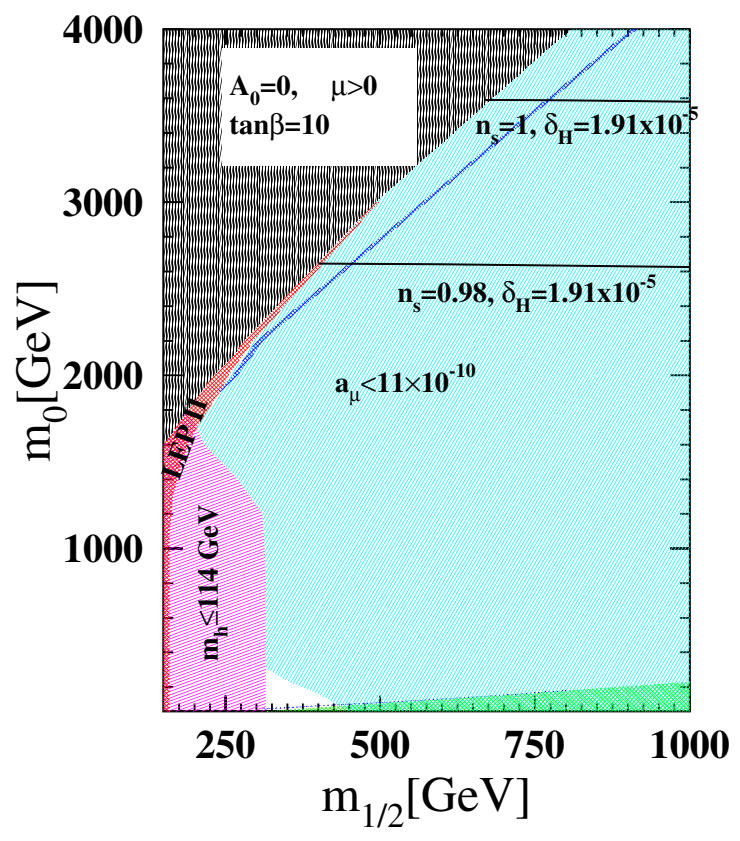

FIG. 5 (color online). The contours for different values of $n_{s}$ and $\delta_{H}$ are shown in the $m_{0}-m_{1 / 2}$ plane for $\tan \beta=10$. We used $\lambda=0.1$ for the contours. We show the dark matter allowed region narrow blue corridor, $g-2$ region (light blue) for $a_{\mu} \leq$ $11 \times 10^{-8}$, Higgs mass $\leq 114 \mathrm{GeV}$ (pink region), and LEPII bounds on SUSY masses (red). The black region is not allowed by radiative electroweak symmetry breaking. We use $m_{t}=$ 172.7 GeV for this graph. 


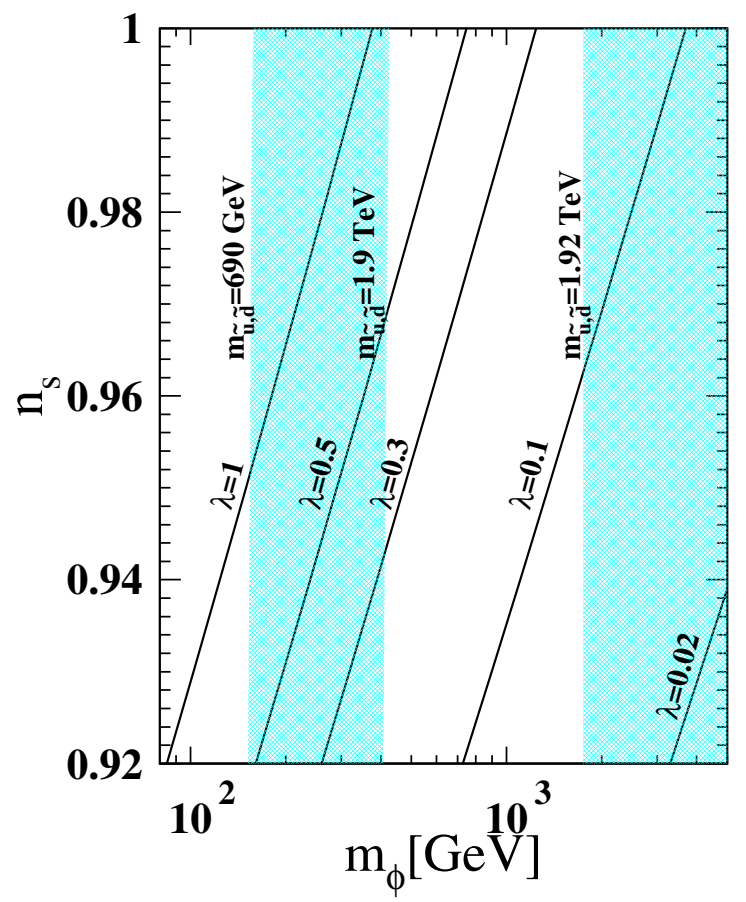

FIG. 6 (color online). Contours of $\lambda$ for $\delta_{H}=1.91 \times 10^{-5}$ in the $n_{s}-m_{\phi}$ plane. The blue band on the left is due to the stauneutralino coannihilation region for $\tan \beta=10$ and the blue band on the right (which continues beyond the plotting range) denotes the focus point region.

two generation squarks masses are $690 \mathrm{GeV}$ and $1.9 \mathrm{TeV}$ for the minimum and maximum values of $m_{\phi}$ allowed by the dark matter and other constraints. The gluino masses for these are $765 \mathrm{GeV}$ and $2.1 \mathrm{TeV}$, respectively. The band is slightly curved due to the shifting of $\phi_{0}$ as a function $\lambda$. (We solve for SUSY parameters from the inflaton mass at $\left.\phi_{0}\right)$. The band on the right which continues beyond the plotting range of the Fig. 6 is due to the Higgsino dominated dark matter. We find that $\lambda$ is mostly $\leq 0.1$ in this region and $m_{\phi}>1.9 \mathrm{TeV}$. In this case the squark masses are much larger than the gluino mass since $m_{0}$ is much larger than $m_{1 / 2}$.

\section{GRAND UNIFIED MODELS AND INCLUSION OF RIGHT-HANDED NEUTRINOS}

\section{A. Embedding MSSM inflation in $S U(5)$ or $S O(10)$ gut}

As we have pointed out, mSUGRA makes a mild assumption that there exists a GUT physics which encompasses MSSM beyond the unification scale $M_{\mathrm{G}} \cdot{ }^{10}$ Here we wish to understand how such embedding would affect inflationary scenario, for instance, would it be possible to

\footnotetext{
${ }^{10} \mathrm{We}$ remind the readers that inflation occurs around a flat direction VEV $\phi_{0} \sim 10^{14} \mathrm{GeV}$. Since $\phi_{0} \ll M_{\mathrm{G}}$, heavy GUT degrees of freedom play no role in the dynamics of MSSM inflation, and hence they can be ignored.
}

single out either LLe or $u d d$ as a candidate for the MSSM inflaton.

The lowest order nonrenormalizable superpotential terms which lift $L L e$ and $u d d$ are [see Eq. (1)]:

$$
\frac{(L L e)^{2}}{M_{\mathrm{P}}^{3}}, \quad \frac{(u d d)^{2}}{M_{\mathrm{P}}^{3}} .
$$

It is generically believed that gravity breaks global symmetries. Then all gauge-invariant terms which are $M_{\mathrm{P}}$ suppressed should appear with $\lambda \sim \mathcal{O}(1)$. Obviously the above terms in Eq. (16) are invariant under the SM. Once the SM is embedded within a GUT at the scale $M_{\mathrm{G}}$, where gauge couplings are unified, the gauge group will be enlarged. Then the question arises whether such terms in Eq. (16) are invariant under the GUT gauge group or not. Note that a GUT singlet is also a singlet under the SM, however, the vice versa is not correct. To answer this question, let us consider $S U(5)$ and $S O(10)$ models separately.

(i) $S U(5)$ : We briefly recollect representations of matter fields in this case: $L$ and $d$ belong to $\overline{\mathbf{5}}$, while $e$ and $u$ belong to 10 of $S U(5)$ group. Thus under $S U(5)$ the superpotential terms in Eq. (16) read

$$
\frac{\overline{\mathbf{5}} \times \overline{\mathbf{5}} \times \mathbf{1 0} \times \overline{\mathbf{5}} \times \overline{\mathbf{5}} \times \mathbf{1 0}}{M_{\mathrm{P}}^{3}} .
$$

This product clearly includes a $S U(5)$ singlet. Therefore in the case of $S U(5)$, we expect that $M_{\mathrm{P}}$ suppressed terms as in Eq. (1) appear with $\lambda \sim \mathcal{O}(1) .^{11}$

(ii) $S O(10)$ : In this case all matter fields of one generation are included in the spinorial representation $\mathbf{1 6}$ of $S O(10)$. Hence the superpotential terms in Eq. (16) are $[\mathbf{1 6}]^{6}$ under $S O(10)$, which does not provide a singlet. A gauge-invariant operator will be obtained by multiplying with a 126-plet Higgs. This implies that in $S O(10)$ the lowest order gauge-invariant superpotential term with 6 matter fields arises at $n=$ 7 level:

$$
\frac{16 \times 16 \times 16 \times 16 \times 16 \times 16 \times 126_{H}}{M_{\mathrm{P}}^{4}} .
$$

Once $\mathbf{1 2 6}_{H}$ acquires a VEV, $S 0(10)$ can break down to a lower ranked subgroup, for instance $S U(5)$. This will induce an effective $n=6$ nonrenormalizable term as in Eq. (1) with

$$
\lambda \sim \frac{\left\langle\mathbf{1 2 6}_{H}\right\rangle}{M_{\mathrm{P}}} \sim \frac{\mathcal{O}\left(M_{\mathrm{GUT}}\right)}{M_{\mathrm{P}}} .
$$

Hence, in the case of $S O(10)$, we can expect $\lambda \sim$ $\mathcal{O}\left(10^{-2}-10^{-1}\right)$ depending on the scale where $S O(10)$ gets broken.

\footnotetext{
${ }^{11}$ If we were to obtain the $(L L e)^{2}$ term by integrating out the heavy fields of the $S U(5)$ GUT, then $\lambda=0$. This is due to the fact that $S U(5)$ preserves $B-L$.
} 
We conclude that embedding MSSM in $S O(10)$ naturally implies $\lambda \ll 1$. Hence an experimental confirmation of the focus point region may be considered as an indication for $S O(10)$. More precise determination of the spectral index $n_{s}$ from future experiments (such as PLANCK) can in addition shed light on the scale of $S O(10)$ breaking. Smaller values of $n_{s}$ (within the range $0.92 \leq n_{s} \leq 1$ ) point to smaller $\lambda$, as can be seen from Fig. 6. This, according to Eq. (19), implies a scale of $S O(10)$ breaking, i.e. $\left\langle\mathbf{1 2 6}_{H}\right\rangle$, which is closer to the GUT scale.

Further note that embedding the MSSM within $S O(10)$ also provides an advantage for obtaining a right-handed neutrino.

\section{B. Including right-handed majorana neutrinos}

Eventually one would need to supplement MSSM with additional ingredients to explain the tiny neutrino masses. Here we consider the most popular framework; the see-saw mechanism which invokes MSSM plus three RH (s)neutrinos $N_{1}, N_{2}, N_{3}$, with respective Majorana masses $M_{i}$. By adding new superfields to MSSM, one can write a larger number of nonrenormalizable gauge-invariant terms of the form in Eq. (1). As a result, a given flat direction might be lifted at a different superpotential level. Then a natural question arises that whether/how adding new superfields will affect the inflaton candidates, i.e. $L L e$ and $u d d$ flat directions.

Since, $N_{i}, 1 \leq i \leq 3$, are SM singlets, we can write the following $n=4$ superpotential terms:

$$
\frac{N_{i} L L e}{M_{\mathrm{P}}}, \quad \frac{N_{i} u d d}{M_{\mathrm{P}}} .
$$

Note that these terms are also singlet under $S U(5)$ and $S O(10)$. In the case of $S U(5)$, the terms in Eq. (20) read $\overline{\mathbf{5}} \times \overline{\mathbf{5}} \times \mathbf{1 0} \times \mathbf{1}$, which includes a singlet. While in the case of $S O(10)$, since $N$ belongs to the 16, the terms in Eq. (20) read $16 \times 16 \times 16 \times 16$, which includes a singlet. Hence both terms in Eq. (20) are allowed in $S U(5)$ or $S O(10)$ embedding of MSSM as well. ${ }^{12}$

We now analyze the case for two flat directions separately.

(i) LLe: First let us consider the LLe flat direction. Taking into account of the family indices, there are 5 independent $D$-flat directions as such [16]. Within MSSM, there are three directions which are $F$ flat at the $n=3$ level, one of which survives until $n=6$. However the term in Eq. (20) leads to three additional $F$-term constraints $F_{N_{i}}=0$, which are more than sufficient to lift the remaining direction at the $n=4$ superpotential level. ${ }^{13}$

\footnotetext{
${ }^{12}$ In the case of $S O(10)$ one can naturally obtain a right-handed neutrino.

${ }^{13}$ The gauge-invariant $L L e$ direction will survive until $n=6$ if all $M_{i} \gg \phi_{0}$. However this is not a phenomenologically viable situation.
}

Generically in this case we would expect $L L e$ to be lifted by a nonrenormalizable operator $n<6$.

(ii) $u d d$ : Next consider the $u d d$ direction. With family indices taken into account, there are 9 independent $D$-flat directions as such [16]. Within MSSM, 3 directions are lifted by $n=4$ terms uude $/ M_{\mathrm{P}}$, while the remaining 6 will be lifted at the $n=6$ level. Note that the superpotential term in Eq. (20) lead to three $F$-term constraints at the $n=4$ level. Nevertheless, 3 directions will still survive until $n=6$.

Based on the above analysis, if we include the $\mathrm{RH}$ neutrinos, we conclude that $u d d$ direction is a more promising inflaton candidate than LLe. The reason is that the flatness of the former will not be lifted in the presence of physically motivated right-handed neutrino fields in addition to that of the MSSM fields.

\section{DISCUSSION AND CONCLUSIONS}

A successful inflation with the right amplitude of the scalar density perturbations, negligible gravity waves and the spectral tilt can be described in the context of MSSM by using the $L L e$ or $u d d$ flat direction as the inflaton. The inflaton mass is constrained from the spectral index and the amplitude of the scalar perturbation. it can be expressed in terms of the squark and slepton masses for $u d d$ and $L L e$ directions, respectively. The constraints on the inflaton mass can then be expressed in terms of the bounds on these masses. These bounds constrain the parameters of the well motivated mSUGRA model.

The parameters of the mSUGRA model are tightly constrained by the dark matter results along with the results from the LEP experiments and the rare decays. After considering all these constraints we have found that an MSSM inflation with a nonrenormalizable coupling $\lambda \sim$ $\mathcal{O}(1)$ (as expected in an effective field theory approach) can be explained in the context of mSUGRA and the stauneutralino coannihilation region is mostly preferred to satisfy the dark matter content of the universe. The SUSY masses of this region are mostly within the reach of the LHC. The maximum value of the gluino mass that is allowed after we include the inflation data along with the dark matter constraint is around $2 \mathrm{TeV}$. We have found that the smaller $\tan \beta$ value allows smaller spectral index which remains within the $2 \sigma$ error of the WMAP data.

Inflation also allows the Higgsino dominated neutralino dark matter, as happens in the focus point region. For this one would require the nonrenormalizable coupling to be $\lambda \sim \mathcal{O}\left(10^{-2}-10^{-1}\right)$, which can be naturally obtained by embedding MSSM in $S O(10)$. Any value of $n_{s}$ in the experimental allowed range can be fit by a suitable choice of $\lambda$. More precise determination of the scalar spectral index in future experiments can in this case shed light on the scale of $S O(10)$ breaking.

We also found that the most promising inflaton candidate is $u d d$. This is due to the fact that the lowest non- 
renormalizable operator which lifts the flat direction remains $n=6$, even if one includes the right-handed Majorana neutrinos. On the other hand $L L e$ can be lifted earlier by $n=4$ superpotential terms.

Thus our analysis provides an example of a standard model gauge-invariant inflaton giving rise to a successful inflation and explains the neutralino CDM abundance, which is in agreement with the present cosmological observations. Moreover this is the first example where the ingredients of a primordial inflation can be put onto test in a laboratory physics such as in the case of LHC.

\section{ACKNOWLEDGMENTS}

We wish to thank Kari Enqvist, Nicolao Fornengo, and Juan Garcia-Bellido for discussions at various stages of this work. The work of R. A. is supported by Perimeter Institute for Theoretical Physics. Research at Perimeter Institute is supported in part by the Government of Canada through NSERC and by the province of Ontario through MRI. A. M. is partly supported by the European Union through Marie Curie Research and Training Network “UNIVERSENET” (MRTN-CT-035863).
[1] R. Allahverdi, K. Enqvist, J. Garcia-Bellido, and A. Mazumdar, Phys. Rev. Lett. 97, 191304 (2006).

[2] R. Allahverdi, A. Kusenko, and A. Mazumdar, hep-ph/ 0608138.

[3] R. Allahverdi and A. Mazumdar, hep-ph/0610069.

[4] R. Allahverdi, K. Enqvist, J. Garcia-Bellido, A. Jokinen, and A. Mazumdar, hep-ph/0610134.

[5] R. Allahverdi, A. Jokinen, and A. Mazumdar, hep-ph/ 0610243.

[6] T. Biswas, R. Brandenberger, A. Mazumdar, and W. Siegel, hep-th/0610274; T. Biswas, A. Mazumdar, and W. Siegel, J. Cosmol. Astropart. Phys. 03 (2006) 009.

[7] G. Lazarides and Q. Shafi, Phys. Lett. B 308, 17 (1993); S. Kasuya, T. Moroi, and F. Takahashi, Phys. Lett. B 593, 33 (2004); A. Jokinen and A. Mazumdar, Phys. Lett. B 597, 222 (2004).

[8] see, http://lhc.web.cern.ch/lhc/.

[9] D. N. Spergel, et al., astro-ph/0603449.

[10] W. H. Kinney, E. W. Kolb, A. Melchiorri, and A. Riotto, Phys. Rev. D 74, 023502 (2006).

[11] R. Allahverdi and A. Mazumdar, J. Cosmol. Astropart. Phys. 10 (2006) 008; hep-ph/0505050; hep-ph/0603244; hep-ph/0608296; M. Postma and A. Mazumdar, J. Cosmol. Astropart. Phys. 01 (2004) 005.

[12] D. Z. Freedman, P. Van Nieuwenhuizen, and S. Ferrara, Phys. Rev. D 13, 3214 (1976); S. Deser and B. Zumino, Phys. Lett. B 62, 335 (1976); A. H. Chamseddine, R. Arnowitt, and P. Nath, Phys. Rev. Lett. 49, 970 (1982).

[13] R. Barbieri, S. Ferrara, and C. A. Savoy, Phys. Lett. B 119, 343 (1982); L. J. Hall, J. D. Lykken, and S. Weinberg, Phys. Rev. D 27, 2359 (1983); P. Nath, R. Arnowitt, and A. H. Chamseddine, Nucl. Phys. B227, 121 (1983); For a review, see H. P. Nilles, Phys. Rep. 110, 1 (1984).

[14] J. Ellis, K. Olive, Y. Santoso, and V. Spanos, Phys. Lett. B 565, 176 (2003); R. Arnowitt, B. Dutta, and B. Hu, hepph/0310103; H. Baer, C. Balazs, A. Belyaev, T.
Krupovnickas, and X. Tata, J. High Energy Phys. 06 (2003) 054; B. Lahanas and D. V. Nanopoulos, Phys. Lett. B 568, 55 (2003); U. Chattopadhyay, A. Corsetti, and P. Nath, Phys. Rev. D 68, 035005 (2003); E. Baltz and P. Gondolo, J. High Energy Phys. 10 (2004) 052; A. Djouadi, M. Drees, and J.L. Kneur, J. High Energy Phys. 03 (2006) 033; J.L. Feng and K. T. Matchev, Phys. Rev. Lett. 86, 3480 (2001).

[15] For reviews, see K. Enqvist and A. Mazumdar, Phys. Rep. 380, 99 (2003); M. Dine and A. Kusenko, Rev. Mod. Phys. 76, 1 (2003).

[16] T. Gherghetta, C. Kolda, and S. P. Martin, Nucl. Phys. B468, 37 (1996).

[17] M. Dine, L. Randall, and S. Thomas, Phys. Rev. Lett. 75, 398 (1995); Nucl. Phys. B458, 291 (1996).

[18] R. Allahverdi, A. R. Frey, and A. Mazumdar, hep-th/ 0701233.

[19] C. P. Burgess, R. Easther, A. Mazumdar, D. F. Mota, and T. Multamaki, J. High Energy Phys. 05 (2005) 067.

[20] J. C. B. Sanchez, K. Dimopoulos, and D. H. Lyth, J. Cosmol. Astropart. Phys. 01 (2007) 015.

[21] A.R. Liddle, D. Parkinson, S. M. Leach, and P. Mukherjee, Phys. Rev. D 74, 083512 (2006).

[22] G. Abbiendi, et al. (LEP Working Group for Higgs Boson Searches and ALEPH, DELPHI, L3, and OPAL Collaborations, Phys. Lett. B 565, 61 (2003).

[23] M. Alam et al., Phys. Rev. Lett. 74, 2885 (1995).

[24] S. Eidelman et al. (Particle Data Group), Phys. Lett. B 592, 1 (2004).

[25] G. Bennett et al. (Muon $g-2$ Collaboration), Phys. Rev. Lett. 92, 161802 (2004); S. Eidelman, ICHEP 2006, Moscow, Russia.

[26] R. Arnowitt et al., hep-ph/0608193; R. Arnowitt, B. Dutta, T. Kamon, N. Kolev, and D. Toback, Phys. Lett. B 639, 46 (2006). 